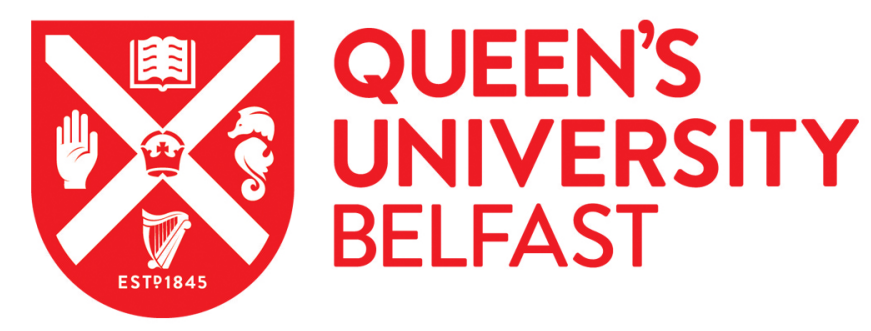

\title{
Towards a sheaf cohomology theory for $\mathrm{C}^{*}$-algebras
}

Mathieu, M. (2020). Towards a sheaf cohomology theory for $C^{*}$-algebras. In Banach Algebras and Applications 2017, Oulu, Finland (pp. 197-212). Walter de Gruyter \& Co., Germany.

Published in:

Banach Algebras and Applications 2017, Oulu, Finland

Document Version:

Peer reviewed version

Queen's University Belfast - Research Portal:

Link to publication record in Queen's University Belfast Research Portal

Publisher rights

Copyright 2019 De Gruyter. This work is made available online in accordance with the publisher's policies. Please refer to any applicable terms of use of the publisher.

\section{General rights}

Copyright for the publications made accessible via the Queen's University Belfast Research Portal is retained by the author(s) and / or other copyright owners and it is a condition of accessing these publications that users recognise and abide by the legal requirements associated with these rights.

Take down policy

The Research Portal is Queen's institutional repository that provides access to Queen's research output. Every effort has been made to ensure that content in the Research Portal does not infringe any person's rights, or applicable UK laws. If you discover content in the Research Portal that you believe breaches copyright or violates any law, please contact openaccess@qub.ac.uk. 


\section{Author??}

Banach Algebras and Applications 2017, Oulu, Finland

De Gruyter Studium

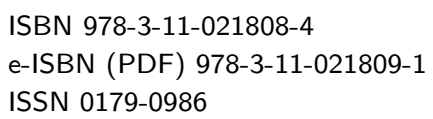

ISBN 978-3-11-021808-4

e-ISBN (PDF) 978-3-11-021809-1

ISSN 0179-0986

\section{Library of Congress Cataloging-in-Publication Data}

A CIP catalog record for this book has been applied for at the Library of Congress.

\section{Bibliographic information published by the Deutsche Nationalbibliothek}

The Deutsche Nationalbibliothek lists this publication in the Deutsche Nationalbibliografie; detailed bibliographic data are available on the Internet at http://dnb.dnb.de.

(C) 2019 Copyright-Text, Walter de Gruyter GmbH, Berlin/Boston

Cover image: Cover-Firma

Typesetting: le-tex publishing services $\mathrm{GmbH}$, Leipzig

Printing and binding: Druckerei $X Y Z$

@ Printed on acid-free paper

Printed in Germany

www.degruyter.com 
Martin Mathieu

\section{Towards a sheaf cohomology theory for $C^{*}$-algebras}

Abstract: In joint work with Pere Ara (Barcelona) we are in the process of developing a full sheaf cohomology theory for noncommutative $C^{*}$-algebras. In this survey, we discuss the difficulties arising from the fact that the appropriate categories of operator module sheaves over sheaves of $C^{*}$-algebras are non-abelian and therefore the homology theory needed has to be set in the more general framework of exact categories.

\section{Keywords:}

\section{Classification:}

\section{Introduction}

Sheaves are ubiquitous in algebraic geometry and algebraic topology. They allow for cohomology theories with 'general coefficient systems' which, to some extent, unify and generalise various other cohomology theories. The invariants that are obtained in this way enable us to differentiate between spaces and capture their properties.

This survey article expands on my talk at the Banach Algebras and Applications conference held in Oulu, Finland in July 2017. In a forthcoming memoir with Pere Ara (Barcelona), [4], we aim to develop a sheaf cohomology theory for general $C^{*}$-algebras; one of a number of obstacles in achieving this goal stems from the absence of appropriate abelian categories. In this paper, the emphasis is on how this obstacle can be overcome. The main change is to exploit the concept an exact categories in the sense of Quillen; we shall follow the survey paper by Bühler [7] in explaining this notion. We introduce the category $\mathcal{O M a d}_{\mathfrak{A}}^{\infty}(X)$ of sheaves of operator modules over $\mathfrak{A}$, where $X$ is a topological space and $\mathfrak{A}$ a sheaf of $C^{*}$-algebras on $X$ and discuss in what way the results (all of which are taken from [4]) have to be modified from analogous situations in categories of algebraic sheaves of modules over sheaves of (commutative) rings as they appear in algebraic geometry, for example.

\section{Topology}

Let us start by reviewing the usual (commutative) topology from a categorical perspective. Let $\mathscr{C}_{1}^{* \text { com }}$ denote the category of unital commutative $C^{*}$-algebras with unital *-homomorphisms as morphisms and

Martin Mathieu , Mathematical Sciences Research Centre, Queen's University Belfast, University Road, Belfast BT7 1NN, Northern Ireland

https://doi.org/10.1515/futur-2012-0001 
let $\mathscr{C} a m p$ be the category of compact Hausdorff spaces with continuous mappings as morphisms. There is a natural equivalence between these two categories given by the following well-known functors. The functor $C: \mathscr{C}$ amp $\longrightarrow \mathscr{C}_{1}^{* \text { com }}$ associates to every compact Hausdorff space $X$ the unital commutative $C^{*}$-algebra $C(X)$ of all complex-valued continuous functions on $X$ and to each continuous function $f: X \rightarrow Y$ into another compact Hausdorff space $Y$ the unital *-homomorphism $C(f): C(Y) \rightarrow C(X)$, $C(f)(g)=g \circ f, g \in C(Y)$. Its inverse is the Gelfand functor $\mathscr{C}_{1}^{* \text { com }} \longrightarrow \mathscr{C}$ omp which assigns to a commutative unital $C^{*}$-algebra $A$ its Gelfand space $\hat{A}$ (the compact Hausdorff space of all multiplicative linear functionals on $A$ ) and to each *-homomorphism $\pi: A \rightarrow B$ of unital commutative $C^{*}$-algebras the continuous function $\hat{\pi}: \hat{B} \rightarrow \hat{A}, \hat{\pi}(\varphi)=\varphi \circ \pi, \varphi \in \hat{B}$.

By means of this, each property of a compact Hausdorff space (which can be expressed within $\mathscr{C} a m p$ ) can be investigated and described in $C^{*}$-algebraic language. Motivated in this way, noncommutative $C^{*}$-algebras can be interpreted as "non-commutative topological spaces" which has been a rather fruitful philosophy leading to many exciting insights in non-commutative geometry in particular. There is, in fact, an analogue of the Gelfand space for any $A \in \mathscr{C}_{1}^{*}$, the category of unital $C^{*}$-algebras with unital *-homomorphisms as morphisms, which we describe now.

The space $\operatorname{Prim}(A)$ of primitive ideals of $A$ with hull-kernel topology consists of the kernels of irreducible representations of $A$, and the open subsets of $\operatorname{Prim}(A)$ can be parametrised by the closed (two-sided) ideals of $A$ as follows. For a topological space $X$, we denote by $\mathcal{U}_{X}$ the category of its open subsets with morphisms given by inclusion. For a closed ideal $I \subseteq A$, the corresponding open subset $U \in \mathscr{U}_{\operatorname{Prim}(A)}$ is given by

$$
U(I)=\{t \in \operatorname{Prim}(A) \mid I \nsubseteq t\} .
$$

Conversely, for each $U \in \mathcal{U}_{\operatorname{Prim}(A)}, A(U)=\bigcap_{t \notin U} t$ defines a closed ideal of $A$. This establishes an order-preserving bijection; that is, $V \subseteq U$ implies $A(V) \subseteq A(U)$. For more details on this, see [12].

Our approach in [4] is partly inspired by algebraic geometry; following Grothendieck's philosophy, varieties are described using the concept of schemes, and a basic notion is the one of a ringed space $\left(X, \mathfrak{O}_{X}\right)$ in which $X$ is the prime spectrum of a commutative unital ring and $\mathfrak{O}_{X}$ is its structure sheaf (of commutative unital rings). See, e.g., [8]. In analogy, we introduce the concept of a " $C^{*}$-ringed space" $(X, \mathfrak{A})$, where $X=\operatorname{Prim}(A)$ for a $C^{*}$-algebra $A$ and $\mathfrak{A}$ is a sheaf of $C^{*}$-algebras on $X$. There are at least two canonical choices for such sheaves, compare [3].

Example 2.1 (The multiplier sheaf). For a $C^{*}$-algebra $A$ with primitive ideal space $\operatorname{Prim}(A)$ let

$$
\mathfrak{M}_{A}: \mathscr{U}_{\operatorname{Prim}(A)} \rightarrow \mathscr{C}_{1}^{*}, \quad \mathfrak{M}_{A}(U)=M(A(U)),
$$

where $M(A(U))$ denotes the multiplier algebra of the closed ideal $A(U)$ of $A$ associated to the open subset $U \subseteq \operatorname{Prim}(A)$, and let $M(A(U)) \rightarrow M(A(V)), V \subseteq U$, denote the restriction homomorphisms.

It is not too diffcult to show that the above functor $\mathfrak{M}_{A}$ defines a sheaf of unital $C^{*}$-algebras [3]. (We shall explain the notion of a sheaf in Section 4 below.) 
Example 2.2 (The injective envelope sheaf). Let $I(B)$ denote the injective envelope of a $C^{*}$-algebra B. We set

$$
\mathfrak{I}_{A}: \mathscr{U}_{\operatorname{Prim}(A)} \rightarrow \mathscr{C}_{1}^{*}, \quad \mathfrak{I}_{A}(U)=p_{U} I(A)=I(A(U)),
$$

where $p_{U}=p_{A(U)}$ denotes the unique central open projection in $I(A)$ such that $p_{A(U)} I(A)$ is the injective envelope of $A(U)$. The restriction morphisms $I(A(U)) \rightarrow I(A(V)), V \subseteq U$, are given by multiplication by $p_{V}\left(\right.$ as $\left.p_{V} \leqslant p_{U}\right)$.

It is fairly easy to verify that this defines a sheaf of unital $C^{*}$-algebras since $\left\{p_{U} \mid U \in \mathcal{U}_{\operatorname{Prim}(A)}\right\}$ is a complete Boolean algebra isomorphic to the Boolean algebra of regular open subsets of $\operatorname{Prim}(A)$, and it is precisely the set of projections of the $A W^{*}$-algebra $Z(I(A))$. For more information on the injective envelope see, e.g., [2].

Before we now move on to study sheaves (of $C^{*}$-algebras and operator modules) in more detail we pause in order to reflect how to overcome a fundamental obstacle arising from the fact that our categories are not abelian, which is a fundamental assumption making homological algebra work in Grothendieck's approach.

\section{Exact categories}

Instead of discussing one of the many equivalent definitions of an abelian category, see, e.g., [10], we focus on some of the decisive features which allow us to work in an abelian category in the same way we do in module categories.

In an abelian category $\mathscr{C}$,

(i) the morphism set between any pair of objects is an abelian group;

(ii) every morphism has a kernel and a cokernel;

(iii) every morphism can be uniquely factorised as

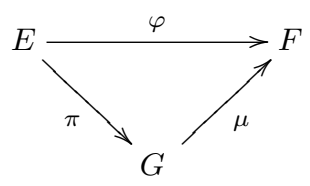

where $\pi$ is an epimorphism and $\mu$ is a monomorphism.

In order to understand why the setting of abelian categories is not the right one for us, let us first of all introduce the notion of an operator module.

Definition 3.1. Let $A$ be a $C^{*}$-algebra. A right $A$-module $E$ which at the same time is an operator space is a right operator $A$-module if it satisfies either of the following equivalent conditions: 
(a) There exist a complete isometry $\Phi: E \longrightarrow B(H, K)$, for some Hilbert spaces $H, K$, and $a *_{-}$ homomorphism $\pi: A \longrightarrow B(H)$ such that $\Phi(x \cdot a)=\Phi(x) \pi(a)$ for all $x \in E, a \in A$.

(b) The bilinear mapping $E \times A \longrightarrow E,(x, a) \mapsto x \cdot$ a extends to a complete contraction $E \otimes_{h} A \longrightarrow E$.

(c) For each $n \in \mathbb{N}, M_{n}(E)$ is a right Banach $M_{n}(A)$-module in the canonical way.

The module $E$ is nondegenerate if the linear span of $\{x \cdot a \mid x \in E, a \in A\}$ is dense in $E$, and it is unital if $A$ is unital and $x \cdot 1=x$ for all $x \in E$.

Our general reference for operator modules is [5], where, for instance, the Haagerup tensor product in the above definition is treated in great detail.

Just as in ring theory, we need to work with categories of modules over a given ring. The categories we must consider (for a $C^{*}$-algebra $A$ ) are:

$\operatorname{OMad}_{A}^{\infty}$

the category with objects the nondegenerate right operator $A$-modules and mor-

phisms the completely bounded $A$-module maps;

$\operatorname{OMOd}_{A}^{1}$

the subcategory of $\mathcal{O M O d}_{A}^{\infty}$ with morphisms the completely contractive $A$ module maps.

While the first of these categories is additive and finitely bicomplete (that is, finite products and sums exist), the second one is non-additive and bicomplete (that is, arbitrary products and coproducts exist). However, none of these categories is abelian!

Instead we will equip these categories with what is termed an "exact structure" (see below); to this end, let us recall some concepts in an arbitrary category $\mathscr{C}$ (which contains a zero object).

Let $f \in \operatorname{Mor}_{\mathscr{C}}(A, B)$ for some $A, B \in \mathscr{C}$.

A morphism $i: K \rightarrow A$ is a kernel of $f$ if $f i=0$ and for each $D \in \mathscr{C}$ and $g \in \operatorname{Mor}_{\mathscr{C}}(D, A)$ with $f g=0$ there is a unique $h \in \operatorname{Mor}_{\mathscr{C}}(D, K)$ making the diagram below commutative

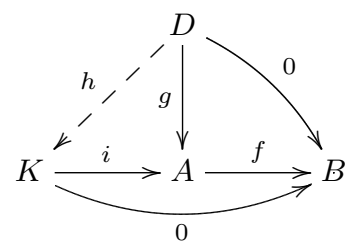

Any kernel is a monomorphism and is, up to isomorphism, unique. We write $i=\operatorname{Ker} f$.

A morphism $p: B \rightarrow C$ is a cokernel of $f$ if $p f=0$ and for each $D \in \mathscr{C}$ and $g \in \operatorname{Mor}_{\mathscr{C}}(B, D)$ with $g f=0$ there is a unique $h \in \operatorname{Mor}_{\mathscr{C}}(C, D)$ making the diagram below commutative

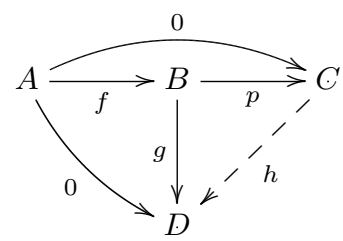


Any cokernel is an epimorphism and is, up to isomorphism, unique. We write $p=\operatorname{Coker} f$.

The situation in both our categories is as follows:

(i) the monomorphisms are injective $A$-module maps;

(ii) the epimorphisms are $A$-module maps with dense range.

However, in

$\operatorname{OMMOd}_{A}^{1}, \quad T: E \rightarrow F$ is a kernel iff is it a complete isometry;

$T: E \rightarrow F$ is a cokernel iff it is a complete quotient map;

while in

$\operatorname{OMOd}_{A}^{\infty}, \quad T: E \rightarrow F$ is a kernel iff it is a completely bounded isomorphism onto its image;

$T: E \rightarrow F$ is a cokernel iff it is surjective and completely open.

This illustrates clearly why the additive category $\mathscr{O} M O d_{A}^{\infty}$ is not abelian.

Homological algebra in abelian categories is based on the concept of a short exact sequence; see, e.g., [11]. For instance, when $E, F, G$ are modules over a ring $R$, exactness of the sequence

$$
0 \longrightarrow E \stackrel{\alpha}{\longrightarrow} F \stackrel{\beta}{\longrightarrow} G \longrightarrow 0
$$

means that $\alpha$ is a monomorphism (so automatically, $\operatorname{ker} \alpha=0$ ), $\beta$ is an epimorphism ( $\operatorname{so} \operatorname{im} \beta=G$ ), and $\operatorname{im} \alpha=\operatorname{ker} \beta$. These conditions can equivalently be summarised by stating that $\alpha$ is the kernel of $\beta$ and $\beta$ is the cokernel of $\alpha$.

In non-abelian categories, such as $\mathcal{G} M{ }^{\circ} d_{A}^{\infty}$, we can re-formulate this key information by introducing so-called "kernel-cokernel pairs"

$$
E \stackrel{M}{\longrightarrow} F \stackrel{P}{\longrightarrow} G
$$

where $E, F, G$ are right operator $A$-modules over the $C^{*}$-algebra $A$ and $M=\operatorname{Ker} P$ and $P=\operatorname{Coker} M$. We can then move on to define an exact category in the sense of Quillen. In an additive category $\mathscr{C}$, a kernel-cokernel pair $(M, P)$ consists of two composable morphisms in $\mathscr{C}$ such that $M=\operatorname{Ker} P$ and $P=\operatorname{Coker} M$,

$$
E_{1}>\stackrel{M}{\longrightarrow} E_{2} \stackrel{P}{\longrightarrow} E_{3}
$$

where $E_{i} \in \mathscr{C}$. A monomorphism $M$ arising in such a pair is called admissible and is denoted as

$$
E>F
$$

and an epimorphism arising in such a pair is called admissible and is denoted as

$$
E \longrightarrow F
$$


Definition 3.2. An exact structure on an additive category $\mathscr{C}$ is a class of kernel-cokernel pairs, closed under isomorphisms, satisfying the following axioms.

$[E 0] \forall E \in \mathscr{C}: 1_{E}$ is an admissible monomorphism;

$\left[E 0^{\mathrm{op}}\right] \forall E \in \mathscr{C}: 1_{E}$ is an admissible epimorphism;

[E1] the class of admissible monomorphisms is closed under composition;

$\left[E 1^{\text {op }}\right]$ the class of admissible epimorphisms is closed under composition;

[E2] the push-out of an admissible monomorphism along an arbitrary morphism exists and yields an admissible monomorphism;

[E2 $\left.{ }^{\mathrm{op}}\right]$ the pull-back of an admissible epimorphism along an arbitrary morphism exists and yields an admissible epimorphism.

Together with an exact structure, $\mathscr{C}$ is called an exact category.

The survey article by Bühler [7] contains a lot of information on exact categories and the way homological algebra is extended from abelian categories to this more general setting.

Definition 3.3. Let $A$ be a $C^{*}$-algebra. We endow the additive category $\mathcal{O M O d}_{A}^{\infty}$ with the exact structure of all kernel-cokernel pairs

$$
E_{1} \stackrel{M}{\longrightarrow} E_{2} \stackrel{P}{\longrightarrow} E_{3}
$$

where $E_{i} \in \mathcal{O M O d}_{A}^{\infty}, 1 \leqslant i \leqslant 3, M$ is a monomorphism in $\mathcal{O M O d}_{A}^{\infty}$ with closed range and completely bounded inverse, $P$ is a completely open mapping in $\mathcal{O M O d}_{A}^{\infty}$ (in particular, surjective) and ker $P=$ $\operatorname{im} M$.

One of the first results in [4] is the following.

Theorem 3.4. Let $A$ be a $C^{*}$-algebra. The class of all kernel-cokernel pairs in $\mathcal{O M O d}_{A}^{\infty}$ is an exact structure on $\mathcal{O M l O d}_{A}^{\infty}$.

This is, however, not sufficient for our purposes as we need to perform arbitrary limits and colimits with operator modules, which will be come clear in the subsequent section. Consequently the exact structure on $\mathcal{O} M \mathrm{Md}_{A}^{\infty}$ needs to induce a similar gadget in the non-additive category $\mathcal{O} \mathscr{M} o d_{A}^{1}$; this is explained in detail in [4].

\section{Sheaves}

In this section we discuss a general framework for sheaves in arbitrary categories (with some small restriction). Throughout, $X$ will be a topological space and $\mathcal{U}_{X}$ the category of open subsets (with open subsets $U$ as objects and $V \rightarrow U$ if and only if $V \subseteq U)$. We assume that $\mathscr{C}$ is a bicomplete 
category with equalisers and coequalisers. This implies in particular the existence of all (small) limits and colimits and the existence of a zero object (and hence zero morphisms); see [10].

Definition 4.1. A presheaf on $X$ in $\mathscr{C}$ is a contravariant functor $\mathfrak{F}: \mathscr{U}_{X} \rightarrow \mathscr{C}$.

A sheaf with values in $\mathscr{C}$ is a presheaf $\mathfrak{F}$ such that $\mathfrak{F}(\varnothing)=0$ and, for every open subset $U$ of $X$ and every open cover $U=\bigcup_{i} U_{i}$, the morphisms $\mathfrak{F}(U) \rightarrow \mathfrak{F}\left(U_{i}\right)$ are the limit of the diagrams $\mathfrak{F}\left(U_{i}\right) \rightarrow$ $\mathfrak{F}\left(U_{i} \cap U_{j}\right)$ for all $i, j$.

In order to explain this definition let us recall the concept of limits in categories. Let $\mathscr{I} \longrightarrow \mathscr{C}$ be a small diagram; we write $E_{i} \in \mathscr{C}$ for the image of an object $i \in \mathscr{I}$ and, if $\varphi_{i j}: i \rightarrow j$ is a morphism, we denote its image by $T_{\varphi_{i j}}: E_{i} \rightarrow E_{j}$. An object $L \in \mathscr{C}$ together with morphisms $\pi_{i}: L \rightarrow E_{i}, i \in \mathscr{I}$ is $a$ limit of the diagram if they make the diagram below commutative and is final with this property.

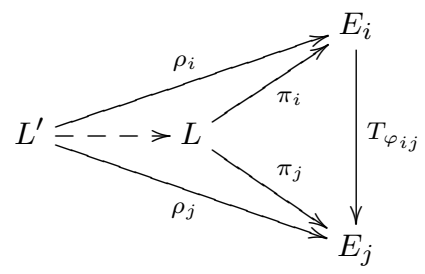

The terminology inverse limits or projective limit is also in use.

Let $U \in \mathscr{U}_{X}$. Let $I$ be a set and $U=\bigcup_{i \in I} U_{i}$ with $U_{i} \in \mathscr{U}_{X}$. Let the index category $I^{(2)}$ consist of unordered pairs of elements in $I$ (we allow the case of singleton sets in $I^{(2)}$ ) where $\{i, j\} \rightarrow\{k, \ell\}$ if $\{i, j\} \subseteq\{k, \ell\}$. Consider the diagram

$$
I^{(2)} \rightarrow\left\{U_{i j}=U_{i} \cap U_{j} \mid\{i, j\} \in I^{(2)}\right\} \subseteq \mathscr{U}_{U}, \quad\{i, j\} \mapsto U_{i j}
$$

composed with the functor $\mathfrak{F}: \mathscr{U}_{X} \rightarrow \mathscr{C}$. Then $\mathfrak{F}(U)$ is the limit of the diagram

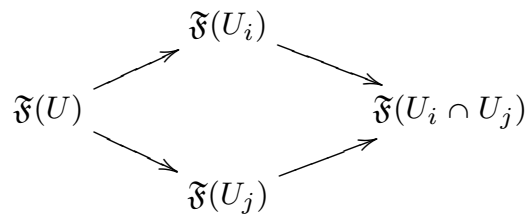

Suppose now that $\mathscr{C}$ is a concrete category, which will be the case in our applications. Then the elements of $\mathfrak{F}(U)$ are called sections over $U \in \mathcal{U}_{X}$. By $s_{\mid V}, V \subseteq U$ open, we mean the "restriction" of $s \in \mathfrak{F}(U)$ to $V$, i.e., the image of $s$ in $\mathfrak{F}(V)$ under the morphism $\rho_{V U}: \mathfrak{F}(U) \rightarrow \mathfrak{F}(V)$. The unique gluing property of a sheaf can be expressed as follows:

for each compatible family of sections $s_{i} \in \mathfrak{F}\left(U_{i}\right)$, i.e., $s_{i \mid U_{i} \cap U_{j}}=s_{j \mid U_{i} \cap U_{j}}$ for all $i, j$, there is a unique section $s \in \mathfrak{F}(U)$ such that $s_{\mid U_{i}}=s_{i}$ for all $i$. 
Let $\mathfrak{F}, \mathfrak{G}$ be (pre)sheaves on $X$. A morphism $\varphi: \mathfrak{F} \rightarrow \mathfrak{G}$ is a natural transformation of the functors, i.e.,

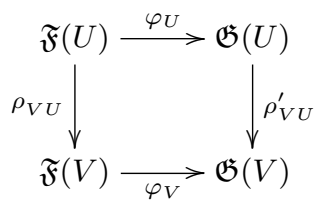

is commutative for all $U, V \in \mathscr{U}_{X}, V \subseteq U$. Hence we have the categories of sheaves on $X, \mathcal{S h}(X, \mathscr{C})$, and of presheaves, $\mathscr{P} \operatorname{Sh}(X, \mathscr{C})$.

Information at a point of $X$ is provided through the stalks. Suppose $\mathfrak{F}$ is a presheaf on $X$, and let $t \in X$. The stalk of $\mathfrak{F}$ at $t$ is defined as $\mathrm{F}_{t}=\underline{\lim }_{\mathscr{U}_{t}} \mathfrak{F}(U)$, where $\mathscr{U}_{t}$ denotes the downward directed family of open neighbourhoods of $t$ and $\lim _{\longrightarrow}$ denotes the (directed) colimit in $\mathscr{C}$.

Here is a first example of how to build a sheaf from a given presheaf. Let $\mathfrak{F}$ be a presheaf on $X$. For each $U \in \mathscr{U}_{X}$, put $\mathfrak{F}^{p}(U)=\prod_{t \in U} \mathrm{~F}_{t}$ and, for $V \subseteq U$, set $\rho_{V U}$ the canonical morphism from $\prod_{t \in U} \mathrm{~F}_{t} \rightarrow \prod_{t \in V} \mathrm{~F}_{t}$. In this way we obtain the product sheaf associated with $\mathfrak{F}$.

We shall assume that the canonical morphism $\sigma_{U}: \mathfrak{F}(U) \rightarrow \mathfrak{F}^{p}(U)$ is a monomorphism whenever $\mathfrak{F}$ is a sheaf.

Local and global information are interrelated via the stalk functor. Suppose $\mathfrak{F}$ and $\mathfrak{G}$ are presheaves on $X$ and that $\varphi: \mathfrak{F} \rightarrow \mathfrak{G}$ is a morphism of presheaves. For each $t \in X$ there is a unique morphism $\varphi_{t}: \mathrm{F}_{t} \rightarrow \mathrm{G}_{t}$ which is defined through the universal property of colimits. In this way, we obtain the stalk functor at $t: \mathscr{P} \operatorname{Sh}(X, \mathscr{C}) \rightarrow \mathscr{C}$.

We observe the following properties:

(a) Let $\varphi^{(1)}, \varphi^{(2)}: \mathfrak{F} \rightarrow \mathfrak{G}$ be morphisms of sheaves. Then $\varphi^{(1)}=\varphi^{(2)}$ if and only if $\varphi_{t}^{(1)}=\varphi_{t}^{(2)}$ for all $t \in X$.

(b) Let $\varphi: \mathfrak{F} \rightarrow \mathfrak{G}$ be a morphism of sheaves. Then

(i) $\varphi$ is a monomorphism if $\varphi_{t}$ is a monomorphism for all $t \in X$;

(ii) $\varphi$ is an epimorphism if $\varphi_{t}$ is an epimorphism for all $t \in X$;

(iii) $\varphi$ is an isomorphism only if $\varphi_{t}$ is an isomorphism for all $t \in X$.

In many algebraic situations, for instance when one considers sheaves of abelian groups, each statement under (b) above is an if-and-only-if statement. This is in general not the case in our analytic situation (e.g., for sheaves of $C^{*}$-algebras), which is one of the causes for headaches.

\section{Our kind of categories}

In this section we introduce the appropriate categories of sheaves of operator modules over sheaves of $C^{*}$ algebras. By $\sigma_{p} p^{1}$ we denote the category of operator spaces with complete contractions as morphisms. 
Definition 5.1. Let $X$ be a topological space, and let $\mathfrak{A}$ be a sheaf of $C^{*}$-algebras on $X$. Suppose $\mathfrak{E}: \mathscr{U}_{X} \longrightarrow \mathcal{O}_{p^{1}}$ is a sheaf in $\mathcal{O} p^{1}$ such that, for each $U \in \mathscr{U}_{X}, \mathfrak{E}(U)$ is a (nondegenerate) right operator $\mathfrak{A}(U)$-module and, whenever $U, V \in \mathscr{U}_{X}$ with $V \subseteq U$, we have that $T_{V U}: \mathfrak{E}(U) \rightarrow \mathfrak{E}(V)$ satisfy

$$
T_{V U}(x \cdot a)=T_{V U}(x) \cdot \pi_{V U}(a) \quad(x \in \mathfrak{E}(U), a \in \mathfrak{A}(U)),
$$

where $\pi_{V U}: \mathfrak{A}(U) \rightarrow \mathfrak{A}(V)$ are the connecting maps in $\mathfrak{A}$. Then we say that $\mathfrak{E}$ is a right operator $\mathfrak{A}$-module on $X$.

In analogy to Section 3 we need to consider the following two categories. Let $\mathfrak{A}$ be a sheaf of $C^{*}$-algebras on the space $X$.

$\mathcal{O} M O d_{\mathfrak{A}}^{\infty}(X), \quad$ the category with objects the right operator $\mathfrak{A}$-modules and morphisms (at $U$ ) the completely bounded $\mathfrak{A}(U)$-module maps;

$\operatorname{OMOd}_{\mathfrak{A}}^{1}(X), \quad$ the subcategory of $\mathcal{O M O}_{\mathfrak{A}}^{\infty}(X)$ with the same objects and morphisms (at $U$ ) the completely contractive $\mathfrak{A}(U)$-module maps.

The same difficulties arise: we need the additive category $\mathcal{O} \mathscr{M} o d_{\mathfrak{A}}^{\infty}(X)$ to be able to apply the methods of homological algebra but we also need the non-additive category $\mathscr{O M}_{\mathscr{O}} \mathrm{d}_{\mathfrak{A}}^{1}(X)$ in order to perform various constructions which are not possible within the bigger category, such as arbitrary products. As none of these categories is abelian, once again we have to define an appropriate exact structure, which will be done in the subsequent section. First, we shall look at various properties of the morphisms.

Let $\mathfrak{E}, \mathfrak{F} \in \mathcal{O M O d}_{\mathfrak{A}}^{\infty}(X)$. A morphism $\varphi: \mathfrak{E} \rightarrow \mathfrak{F}$ is a natural transformation between the functors $\mathfrak{E}$ and $\mathfrak{F}$. In $\mathcal{O} \mathscr{M} o d_{\mathfrak{A}}^{\infty}(X)$, this means we have a commutative diagram

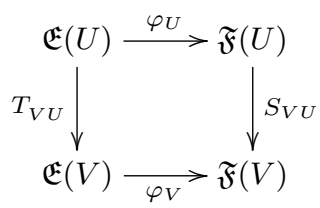

for each $U \in \mathscr{U}_{X}$, where each $\varphi_{U}$ is a completely bounded $\mathfrak{A}(U)$-module map and $\|\varphi\|_{c b}:=$ $\sup _{U \in \mathscr{U}_{X}}\left\|\varphi_{U}\right\|_{c b}<\infty$. In $\mathcal{O} M O d_{\mathfrak{A}}^{1}(X)$ we require, in addition, $\|\varphi\|_{c b} \leqslant 1$. These morphism sets shall be denoted by $C B_{\mathfrak{A}}(\mathfrak{E}, \mathfrak{F})$ and $C B_{\mathfrak{A}}^{1}(\mathfrak{E}, \mathfrak{F})$, respectively.

The stalk functor in these categories is generally well behaved.

Proposition 5.1. Let $\mathfrak{E}$ be a presheaf of operator $\mathfrak{A}$-modules on $X$. For each $t \in X$, the stalk $\mathrm{E}_{t}$ belongs to $\operatorname{OMOd}_{\mathrm{A}_{t}}^{1}$.

Proposition 5.2. Let $\mathfrak{E}, \mathfrak{F}$ be presheaves of operator $\mathfrak{A}$-modules on $X$ and let $\varphi \in C B_{\mathfrak{A}}(\mathfrak{E}, \mathfrak{F})$. For each $t \in X$, we have $\varphi_{t} \in C B_{\mathrm{A}_{t}}\left(\mathrm{E}_{t}, \mathrm{~F}_{t}\right)$. Moreover, $\varphi$ is completely contractive if and only if $\varphi_{t}$ is completely contractive for all $t \in X$. 
To describe the isomorphisms, however, is more delicate than for sheaves of purely algebraic structures; compare, e.g., [9].

Theorem 5.2. Let $\varphi \in C B_{\mathfrak{A}}(\mathfrak{E}, \mathfrak{F})$ for some $\mathfrak{E}, \mathfrak{F} \in \mathcal{O M M O d}_{\mathfrak{A}}^{\infty}(X)$. Then the following conditions are equivalent.

(a) $\varphi$ is an isomorphism;

(b) $\varphi_{U}$ is an isomorphism in $\mathcal{O M O d}_{\mathfrak{A}(U)}^{\infty}$ for each $U \in \mathcal{U}_{X}$ and $\sup _{U \in \mathcal{U}_{X}}\left\|\varphi_{U}^{-1}\right\|_{c b}<\infty$;

(c) $\varphi_{t}$ is an isomorphism in $\mathcal{O M N O d}_{\mathrm{A}_{t}}^{\infty}$ for each $t \in X, \sup _{t \in X}\left\|\varphi_{t}^{-1}\right\|_{c b}<\infty$ and $\varphi_{t}^{0}$ is surjective for each $t \in X$.

Here, $\varphi_{t}^{0}: \mathrm{E}_{t}^{0} \rightarrow \mathrm{F}_{t}^{0}$ is the restriction of $\varphi_{t}$ to the uncompleted directed colimit which is given by $\mathrm{E}_{t}^{0}=\bigcup_{U \in \mathscr{U}_{t}} T_{U} \mathfrak{E}(U)$, where $T_{U}: \mathfrak{E}(U) \rightarrow \mathrm{E}_{t}, U \in \mathscr{U}_{t}$ are the canonical maps into the colimit.

\section{$6 \operatorname{OMOd}_{\mathfrak{A}}^{\infty}(X)$ is exact}

Our task here is to define an exact structure on $\mathcal{O M M O d}_{\mathfrak{A}}^{\infty}(X)$. Therefore, we need to define and understand the kernel and the cokernel of a morphism in $\mathcal{O M}_{\mathfrak{A}} \mathrm{d}_{\mathfrak{A}}^{\infty}(X)$.

Definition 6.1. Let $\varphi \in C B_{\mathfrak{A}}(\mathfrak{E}, \mathfrak{F})$ for some $\mathfrak{E}, \mathfrak{F} \in \mathcal{O M M O d}_{\mathfrak{A}}^{\infty}(X)$. For each $U \in \mathcal{U}_{X}$, we set $(\operatorname{Ker} \varphi)(U)=\operatorname{ker} \varphi_{U}$.

Since $T_{V U} \operatorname{ker} \varphi_{U} \subseteq \operatorname{ker} \varphi_{V}$ whenever $V \subseteq U$, we can restrict the connecting morphisms $T_{V U}$ in $\mathfrak{E}$ to connecting morphisms $\operatorname{ker} \varphi_{U} \rightarrow \operatorname{ker} \varphi_{V}$. In this way, we obtain a sub-presheaf $\operatorname{Ker} \varphi$ of $\mathfrak{E}$ which is easily checked to be a sheaf.

We will call this the sheaf kernel or simply the kernel of $\varphi$ and denote it by $\operatorname{Ker} \varphi$.

Definition 6.2. Let $\varphi \in C B_{\mathfrak{A}}(\mathfrak{E}, \mathfrak{F})$ for some $\mathfrak{E}, \mathfrak{F} \in \mathcal{O M M O d}_{\mathfrak{A}}^{\infty}(X)$. For each $U \in \mathcal{U}_{X}$, we set $(\operatorname{PCoker} \varphi)(U)=\operatorname{coker} \varphi_{U}$. Recall that $\operatorname{coker} \varphi_{U}=\mathfrak{F}(U) / \overline{\operatorname{im} \varphi_{U}}$ by definition.

Since $S_{V U} \operatorname{im} \varphi_{U} \subseteq \operatorname{im} \varphi_{V}$ whenever $V \subseteq U$, the connecting morphisms $S_{V U}$ in $\mathfrak{F}$ induce connecting morphisms coker $\varphi_{U} \rightarrow \operatorname{coker} \varphi_{V}$. In this way, we obtain a presheaf PCoker $\varphi$, called the presheaf cokernel of $\varphi$.

Since this is in general not a sheaf, we define the sheaf cokernel or simply the cokernel of $\varphi$ as the sheafification of $\operatorname{PCoker} \varphi$ : Coker $\varphi=(\operatorname{PCoker} \varphi)^{\sim}$.

Many constructions in sheaf theory initially lead to a presheaf rather than a sheaf. This is, for example, the case in Definition 6.2 above. The process of sheafification associates to a given presheaf a sheaf, unique up to isomorphism, which has the same stalks and with the universal property that every morphism $\varphi$ from the presheaf into another sheaf 'extends' to a morphism $\tilde{\varphi}$ defined on the sheafification such that $\varphi_{t}=\tilde{\varphi}_{t}$ for all $t \in X$. This is a fundamental device which is well used in sheaves of abelian 
groups, e.g. In our setting, it has to be modified in order to cater for the analytic structures; see [4, Section 6].

Remarks 6.1. 1. The canonical embedding $\iota: \operatorname{Ker} \varphi \rightarrow \mathfrak{E}$ is in fact a monomorphism in $\mathfrak{O M M d}_{\mathfrak{A}}^{1}(X)$ since each $\iota_{U}$ is a complete isometry.

2. The canonical quotient morphism $\pi: \mathfrak{F} \rightarrow \operatorname{PCoker} \varphi$ is in fact an epimorphism in $\mathcal{O M M O d}_{\mathfrak{A}}^{1}(X)$ since each $\pi_{U}$ is a completely contractive complete quotient mapping.

Letting $\tilde{\pi}: \mathfrak{F} \rightarrow$ Coker $\varphi$ be given by $\tilde{\pi}_{U}=\eta_{U} \pi_{U}, U \in \mathcal{U}_{X}$, where $\eta$ is the sheafification transformation, we have $\|\tilde{\pi}\|_{c b} \leqslant 1$, since $\left\|\tilde{\pi}_{U}\right\|_{c b} \leqslant\left\|\eta_{U}\right\|_{c b}\left\|\pi_{U}\right\|_{c b}$ for all $U \in \mathcal{U}_{X}$.

Theorem 6.3. Let $X$ be a topological space and let $\mathfrak{A}$ be a sheaf of $C^{*}$-algebras on $X$. Let $\mathfrak{E}, \mathfrak{F} \in$ $\operatorname{OMOd}_{\mathfrak{A}}^{\infty}(X)$. For each $\varphi \in C B_{\mathfrak{A}}(\mathfrak{E}, \mathfrak{F}), \operatorname{Ker} \varphi$ is a kernel in the category $\mathcal{O M O d}_{\mathfrak{A}}^{\infty}(X)$ and Coker $\varphi$ is a cokernel in $\mathcal{O M O d}_{\mathfrak{A}}^{\infty}(X)$. In particular, the morphisms $\iota$ and $\tilde{\pi}$ defined above are a monomorphism and an epimorphism, respectively in $\mathcal{O M O d}_{\mathfrak{A}}^{\infty}(X)$.

I will try to give you a glimpse into the proof of the above theorem. Suppose $\psi \in C B_{\mathfrak{A}}(\mathfrak{G}, \mathfrak{E})$ for some $\mathfrak{G} \in \mathcal{O M O d}_{\mathfrak{A}}^{\infty}(X)$ is such that $\varphi \psi=0$. For each $U \in \mathcal{U}_{X}$, we have the following commutative diagram

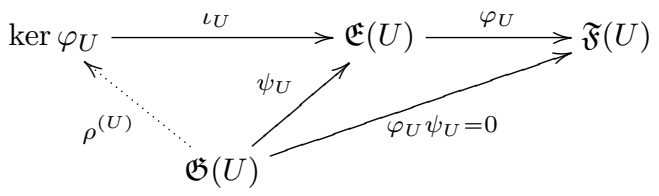

where $\rho^{(U)} \in C B_{\mathfrak{A}(U)}\left(\mathfrak{G}(U), \operatorname{ker} \varphi_{U}\right)$ is the unique morphism such that $\iota_{U} \rho^{(U)}=\psi_{U}$; this follows form the fact that $\operatorname{ker} \varphi_{U}$ is a kernel in $\mathfrak{O M}_{\mathfrak{O}} \boldsymbol{d}_{\mathfrak{A}(U)}^{\infty}$. See Section 3 above. In order to assemble $\left\{\rho^{(U)}\right\}$ to a morphism in $\mathcal{O M O d}_{\mathfrak{A}}^{\infty}(X)$, let $V \in \mathcal{U}_{X}, V \subseteq U$ and denote the connecting morphisms in $\mathfrak{E}, \mathfrak{F}$ and $\mathfrak{G}$, respectively, by $T_{V U}, S_{V U}$ and $R_{V U}$, respectively. One then has to work through the diagram below

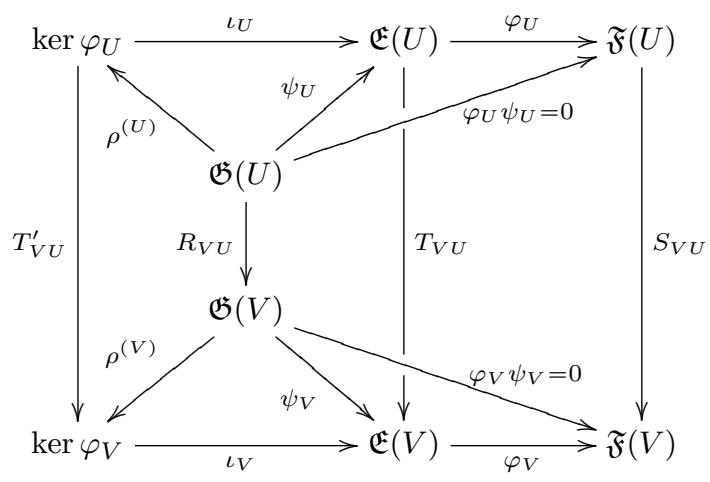

in order to obtain the unique morphism $\rho \in C B_{\mathfrak{A}}(\mathfrak{G}, \operatorname{Ker} \varphi)$ such that $\iota \rho=\psi$. It follows that $\operatorname{Ker} \varphi$ is a kernel in $\mathfrak{O M O d}_{\mathfrak{A}}^{\infty}(X)$. 
Remark 6.1. Let $\mathscr{C}$ be a category with the property that every morphism has both a kernel and a cokernel (which is the case in $\mathscr{G M O d}_{\mathfrak{A}}^{\infty}(X)$ and in $\mathscr{O} \mathscr{M} o d_{\mathfrak{A}}^{1}(X)$ ). Then a morphism which is a kernel necessarily is the kernel of its cokernel, and a morphism which is a cokernel necessarily is the cokernel of its kernel.

We have the following characterisations of kernels and cokernels in $\mathcal{O} M \mathscr{O} \mathbb{d}_{\mathfrak{A}}^{\infty}(X)$, where the superscript ${ }^{0}$ indicates the induced mapping on the uncompleted direct limit; compare the remark after Theorem 5.2.

Proposition 6.1. Let $\mu \in C B_{\mathfrak{A}}(\mathfrak{E}, \mathfrak{F})$ for some $\mathfrak{E}, \mathfrak{F} \in \mathcal{O M l O d}_{\mathfrak{A}}^{\infty}(X)$ and let $\tilde{\pi}: \mathfrak{F} \rightarrow$ Coker $\mu$. Then $\mu$ is a kernel in $\mathcal{O M O d}_{\mathfrak{A}}^{\infty}(X)$ if and only if, for each $t \in X,\left(\mu_{t}, \pi_{t}\right)$ is a kernel-cokernel pair in $\mathcal{O M M O d}_{\mathrm{A}_{t}}^{\infty}$ with $\operatorname{im} \mu_{t}^{0}=\operatorname{ker} \pi_{t}^{0}$ and $\sup _{t \in X}\left\|\mu_{t}^{-1}\right\|_{c b}<\infty$.

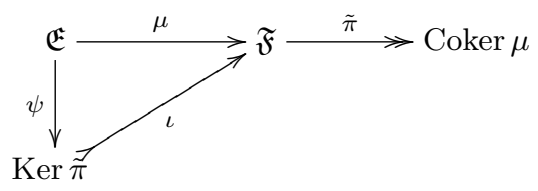

Proposition 6.2. Let $\varpi \in C B_{\mathfrak{A}}(\mathfrak{F}, \mathfrak{G})$ for some $\mathfrak{F}, \mathfrak{G} \in \operatorname{OMMOd}_{\mathfrak{A}}^{\infty}(X)$. Then $\varpi$ is a cokernel in $\mathcal{O M O d}_{\mathfrak{A}}^{\infty}(X)$ if and only if, for each $t \in X, \varpi_{t}^{0}$ is completely open onto $\mathrm{G}_{t}^{0}$ and $\sup _{t \in X}\left\|\varpi_{t}^{-1}\right\|_{c b}<\infty$.

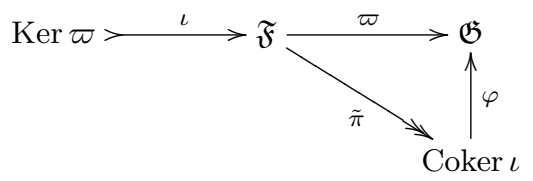

Definition 6.4. Let $X$ be a topological space and let $\mathfrak{A}$ be a sheaf of $C^{*}$-algebras on $X$. Let $\mathscr{E} x_{\mathfrak{A}}(X)$ denote the collection of all kernel-cokernels pairs in $\mathcal{O M O d}_{\mathfrak{A}}^{\infty}(X)$

$$
\mathfrak{E}_{1}>\stackrel{\mu}{\longrightarrow} \mathfrak{E}_{2} \stackrel{\varpi}{\longrightarrow} \mathfrak{E}_{3} \text {. }
$$

We call this the canonical exact structure on $\mathcal{O M O d}_{\mathfrak{A}}^{\infty}(X)$, which is justified by the following result.

Theorem 6.5. The class $\mathscr{E} x_{\mathfrak{A}}(X)$ of all kernel-cokernel pairs defines an exact structure on $\mathcal{O M}_{M} \mathbb{d}_{\mathfrak{A}}^{\infty}(X)$.

Remarks 6.2. 1. $\mathscr{E}_{\mathfrak{A}}(X)$ is the largest exact structure on $\mathcal{O M}_{\mathfrak{A}} \mathrm{d}_{\mathfrak{A}}^{\infty}(X)$.

2. Axioms [E0] and [E0 $\left.{ }^{\mathrm{OP}}\right]$ are easily verified, the others are hard.

3. Given a kernel-cokernel pair $(\mu, \varpi)$ in $\mathscr{E}_{x_{\mathfrak{A}}}(X)$ we obtain an isomorphic pair $(\iota, \tilde{\pi})$

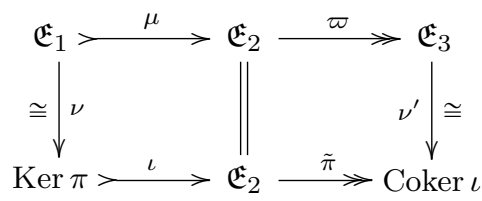


and for each $U \in \mathcal{U}_{X}$, we get an exact sequence in $\mathcal{O M}_{\mathfrak{M}} \mathbf{d}_{\mathfrak{A}(U)}^{1}$

$$
0 \longrightarrow \operatorname{ker} \pi_{U} \stackrel{\iota_{U}}{\longrightarrow} \mathfrak{E}_{2}(U) \stackrel{\pi_{U}}{\longrightarrow} \operatorname{coker} \iota_{U} \longrightarrow 0
$$

where coker $\iota_{U}=\mathfrak{E}_{2}(U) / \mathrm{im} \iota_{U}$ since $\iota_{U}$ is a complete isometry.

\section{Homological Algebra in $\operatorname{GMOd}_{\mathfrak{A}}^{\infty}(X)$}

The short exact sequences that are used in abelian categories are replaced in $\mathscr{E}_{\mathfrak{A}}(X)$ by kernel-cokernel pairs

$$
\mathfrak{E}_{1}>^{\mu}>\mathfrak{E}_{2} \stackrel{\varpi}{\longrightarrow} \mathfrak{E}_{3}
$$

where $\mathfrak{E}_{i} \in \mathcal{O} M O d_{\mathfrak{A}}^{\infty}(X)$, which we also address as short exact sequences. To make the homological algebra work, we have to restrict the morphisms we can use.

Definition 7.1. The morphism $\varphi \in C B_{\mathfrak{A}}(\mathfrak{E}, \mathfrak{F}), \mathfrak{E}, \mathfrak{F} \in \mathcal{O M M O d}_{\mathfrak{A}}^{\infty}(X)$ is called admissible if it can be factorised as

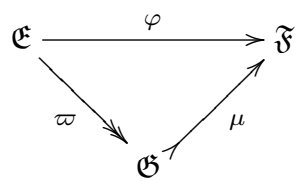

for some admissible monomorphism $\mu$ and some admissible epimorphism $\varpi$ in $\mathcal{O M}_{\mathfrak{M}} \mathrm{d}_{\mathfrak{A}}^{\infty}(X)$.

Definition 7.2. A sequence of admissible morphisms in $\mathcal{O M O d}_{\mathfrak{A}}^{\infty}(X)$

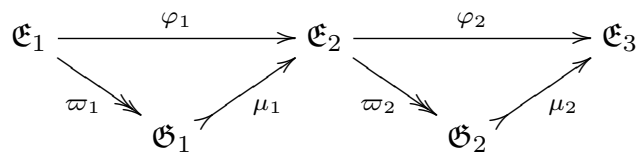

is said to be exact if the short sequence $\mathfrak{G}_{1} \stackrel{\mu_{1}}{\longrightarrow} \mathfrak{E}_{2} \stackrel{\varpi_{2}}{\longrightarrow} \mathfrak{G}_{2}$ is exact. An arbitrary sequence of admissible morphisms in $\mathfrak{O M O d}_{\mathfrak{A}}^{\infty}(X)$ is exact if the sequences given by any two consecutive morphisms are exact.

We can characterise the admissible morphisms using the stalks as follows.

Theorem 7.3. Let $\varphi \in C B_{\mathfrak{A}}(\mathfrak{E}, \mathfrak{F})$ for some $\mathfrak{E}, \mathfrak{F} \in \mathcal{O M M O d}_{\mathfrak{A}}^{\infty}(X)$. Then $\varphi$ is admissible if and only if, for each $t \in X$,

(i) $\varphi_{t} \in C B_{\mathrm{A}_{t}}\left(\mathrm{E}_{t}, \mathrm{~F}_{t}\right)$ is admissible;

(ii) $\hat{\varphi}_{t}^{0}$ is surjective; 
(iii) $(\operatorname{Ker} \varphi)_{t}=\operatorname{ker} \varphi_{t},(\operatorname{Coker} \varphi)_{t}=\operatorname{coker} \varphi_{t}$, and

(iv) $\sup _{t \in X}\left\|\varphi_{t}^{-1}\right\|_{c b}<\infty$.

In particular, the stalk functor at $t \in X$ is exact from $\mathscr{O} \mathscr{M} o d_{\mathfrak{A}}^{\infty}(X)$ to $\mathscr{O} \mathscr{M} o d_{\mathrm{A}_{t}}^{\infty}$. This result uses the following factorisation of admissible morphisms which relies on the fact that $\mathcal{O M M O d}_{\mathfrak{A}}^{\infty}(X)$ is semi-abelian. (In an abelian category, $\hat{\varphi}$ below is always an isomorphism.)

Proposition 7.1. For every morphism $\varphi \in C B_{\mathfrak{A}}(\mathfrak{E}, \mathfrak{F})$, there exists a unique factorisation $\varphi=\mu \hat{\varphi} \pi$ as given in the commutative diagram below.

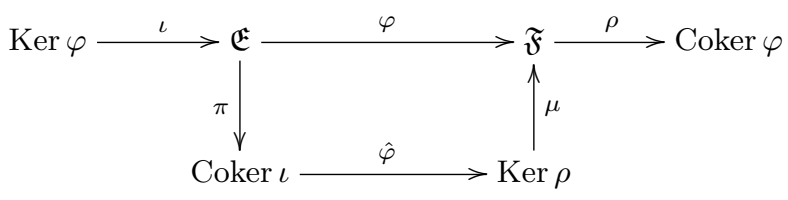

Moreover, $\varphi$ is admissible if and only if $\hat{\varphi}$ is an isomorphism.

The next steps will then include introducing the cohomology groups as the right derived functors of the global section functor applied to an injective resolution of a sheaf of operator modules $\mathfrak{F} \in \mathcal{O} M M_{0} d_{\mathfrak{A}}^{\infty}(X)$. To this end, we build injective sheaves using the injective envelope of an operator module from which it follows that $\mathcal{O} \mathscr{M} a d_{\mathfrak{A}}^{\infty}(X)$ has enough injectives. The homological algebra provided by the exact structure is essential to this. 


\section{Bibliography}

[1] P. Ara and M. Mathieu, Local multipliers of $C^{*}$-algebras, Springer-Verlag, London, 2003.

[2] P. Ara And M. Mathieu, Maximal $C^{*}$-algebras of quotients and injective envelopes of $C^{*}$-algebras, Houston J. Math. 34 (2008), 827-872.

[3] P. Ara and M. Mathieu, Sheaves of $C^{*}$-algebras, Math. Nachr. 283 (2010), 21-39.

[4] P. Ara And M. Mathieu, Sheaf cohomology for $C^{*}$-algebras, memoir in preparation.

[5] D. P. Blecher and Ch. Le Merdy, Operator algebras and their modules. An operator space approach, London Math. Soc. Monographs 30, Claredon Press, Oxford, 2004.

[6] G. E. Bredon, Sheaf theory, Graduate Texts in Maths. 170, Springer-Verlag, New York, 2nd. ed., 1997.

[7] Th. BühleR, Exact categories, Expo. Math. 28 (2010), 1-69.

[8] R. Hartshorne, Algebraic geometry, Springer-Verlag, New York, 1997.

[9] B. Iversen, Cohomology of sheaves, Springer-Verlag, Berlin, 1986.

[10] S. MacLane, Categories for the working mathematician, Graduate Texts in Maths. 5, SpringerVerlag, New York, 2nd ed., 1998.

[11] M. S. Osborne, Basic homological algebra, Graduate Texts in Maths. 196, Springer-Verlag, New York, 2nd ed., 2000.

[12] G. K. Pedersen, $C^{*}$-algebras and their automorphism groups, Academic Press, London, 1979. 